\title{
PRODUCTION AND RECOIL LOSS OF COSMOGENIC NUCLIDES IN PRESOLAR GRAINS
}

\author{
Reto TrapPitsch ${ }^{1}$ and INGo Leya ${ }^{2}$ \\ ${ }^{1}$ Department of the Geophysical Sciences and Chicago Center for Cosmochemistry, The University of Chicago, Chicago, IL 60637, USA; trappitsch@uchicago.edu \\ ${ }^{2}$ Space Research and Planetary Sciences, University of Bern, Bern, 3012, Switzerland; ingo.leya @ space.unibe.ch \\ Received 2015 December 23; accepted 2016 April 12; published 2016 May 16
}

\begin{abstract}
Presolar grains are small particles that condensed in the vicinity of dying stars. Some of these grains survived the voyage through the interstellar medium (ISM) and were incorporated into meteorite parent bodies at the formation of the Solar System. An important question is when these stellar processes happened, i.e., how long presolar grains were drifting through the ISM. While conventional radiometric dating of such small grains is very difficult, presolar grains are irradiated with galactic cosmic rays (GCRs) in the ISM, which induce the production of cosmogenic nuclides. This opens the possibility to determine cosmic-ray exposure (CRE) ages, i.e., how long presolar grains were irradiated in the ISM. Here, we present a new model for the production and loss of cosmogenic ${ }^{3} \mathrm{He},{ }^{6,7} \mathrm{Li}$, and ${ }^{21,22} \mathrm{Ne}$ in presolar $\mathrm{SiC}$ grains. The cosmogenic production rates are calculated using a state-of-the-art nuclear cross-section database and a GCR spectrum in the ISM consistent with recent Voyager data. Our findings are that previously measured ${ }^{3} \mathrm{He}$ and ${ }^{21} \mathrm{Ne}$ CRE ages agree within the (sometimes large) $2 \sigma$ uncertainties and that the CRE ages for most presolar grains are smaller than the predicted survival times. The obtained results are relatively robust since interferences from implanted low-energy GCRs into the presolar SiC grains and/or from cosmogenic production within the meteoroid can be neglected.
\end{abstract}

Key words: cosmic rays - dust, extinction - meteorites, meteors, meteoroids - nuclear reactions, nucleosynthesis, abundances

\section{INTRODUCTION}

Primitive meteorites contain microscopic grains of stardust that condensed either in the expanding envelopes of red-giant branch and/or asymptotic giant branch (AGB) stars or in the ejecta of supernovae. Some of the so-called presolar grains survived travel in the interstellar medium (ISM) and were finally incorporated into the molecular cloud from which the Solar System formed. Since presolar grains predate Solar System formation, they provide the oldest material that can be analyzed in the laboratory and thereby offer a direct link between astrophysics, cosmochemistry, and meteorite research (e.g., Bernatowicz \& Zinner 1997; Ott 2001). The presolar grain types discovered so far include $\mathrm{C}$-rich phases, e.g., diamonds, $\mathrm{SiC}$, graphite, O-rich phases, e.g., $\mathrm{Al}_{2} \mathrm{O}_{3}, \mathrm{MgAl}_{2} \mathrm{O}_{4}$, silicates, and nitrides, e.g., $\mathrm{Si}_{3} \mathrm{~N}_{4}$. For a review see, e.g., Zinner (2014, pp. 181-213). Isotopic studies of presolar grains provide valuable information on stellar processes. However, an important question thereby is when these stellar processes happened, i.e., what was the time between condensation of presolar grains and their incorporation into the early Solar System. Conventional radiometric dating is difficult, if not impossible, not only due to the small grain size but predominantly due to the large isotopic abundance anomalies in almost all elements (e.g., Meyer \& Zinner 2006, pp. 69-108), which makes a reliable determination of initial parent and daughter nuclide abundances impossible. As an alternative Tang \& Anders (1988a, 1988b) proposed to study cosmic-ray exposure (CRE) ages of the grains, i.e., the time the grains traveled through the ISM before their incorporation into the early Solar System.

The destruction of dust grains in the ISM is assumed to be be due to interactions with supernova shock waves (e.g., Jones et al. 1994, 1997) with calculated lifetimes of $\sim 600 \mathrm{Ma}$ for graphite grains and $\sim 400 \mathrm{Ma}$ for silicate grains (e.g., Jones et al. 1997). The expected lifetimes for $\mathrm{SiC}$ are up to $\sim 1.5 \mathrm{Ga}$
(Bernatowicz et al. 2003). Though the lifetimes are relatively long, they are significantly shorter than the timescale on which stellar dust is replenished. It is therefore possible that some of the grains found in meteorites have their origin in a late stellar event, i.e., a stellar event that occurred shortly before solar system formation. It has also been speculated that the stellar event, i.e., the supernova (e.g., Cameron \& Truran 1977) or AGB star (Foster \& Boss 1996, 1997), might have triggered the formation of the Solar System. Alternatively, the presolar grains now selected from meteorites could have been grown in the ISM itself by direct condensation out of the gas, in accordance with the observation that the gas in the ISM is depleted in refractory elements. Clearly, to distinguish between both scenarios and to better understand the history of presolar grains a proper knowledge of their lifetime is necessary. A possibility thereby is to determine their CRE ages.

The first study of CRE ages for presolar $\mathrm{SiC}$ grains yielded surprisingly low ages in the range $\sim 10$ to $\sim 130 \mathrm{Ma}$, with ages increasing with the average grain size (Lewis et al. 1990, 1994). Later, Ott \& Begemann (2000) experimentally demonstrated that presolar grains very often lost essentially all of its spallogenic neon due to recoil. They concluded that, first, using spallogenic ${ }^{21} \mathrm{Ne}$ for determining CRE ages for presolar grains is difficult and, second, that spallogenic xenon could be more reliable because of lower recoil losses. In subsequent studies, Mohapatra et al. (2001) and Ott et al. (2001) experimentally determined, among others, that recoil losses for xenon isotopes are indeed $\sim 10$ times lower than for neon isotopes. They also argued that the production of xenon is less dependent on the galactic cosmic-ray (GCR) spectrum, which is also an advantage because the GCR particle spectrum in the ISM is not well known. Based on these studies and by using the xenon data from Lewis et al. (1994), Ott et al. (2005) determined surprisingly low CRE ages of a few tens of Ma for most of the grain size separates, i.e., again 
considerably shorter than expected lifetimes. Some of the ages were as high as $175 \mathrm{Ma}$. However the preferred interpretation by Ott et al. (2005) was that ages were short, probably less than $20 \mathrm{Ma}$. However, interpretation of the data was made difficult due to ill-known production rates and due to a trapped xenon component. Note that the choice of the trapped component had an influence on the determined ages.

Another approach that avoids problems caused by trapped components, which compromises CRE age studies based on noble gases, was used by Gyngard et al. (2009a, 2009b) by studying the lithium isotopic composition in presolar $\mathrm{SiC}$ grains larger than $5 \mu \mathrm{m}$. Since indigenous lithium concentrations in $\mathrm{SiC}$ are low, a cosmogenic lithium signal in the form of ${ }^{6} \mathrm{Li}$ excesses is detectable in some grains. Note, however, that the non-cosmogenic lithium concentration of a typical presolar $\mathrm{SiC}$ grain is still about two orders of magnitude higher than the non-cosmogenic neon concentration. In contrast, the production rates for lithium isotopes are only a factor of about two higher than the ones for neon production. However, the advantage of using lithium over the noble gases is that the star itself is unlikely to add any lithium to the presolar grain. Hence, the isotopic composition of the background lithium can be assumed to be solar. Since ${ }^{6} \mathrm{Li}$ and ${ }^{7} \mathrm{Li}$ are light isotopes, recoil effects are again significant and must be corrected for. Doing so the authors obtained exposure ages in the range $\sim 40 \mathrm{Ma}-\sim 1 \mathrm{Ga}$, i.e., more in accord with theoretical expectations of interstellar grain lifetimes. By using spallogenic helium and neon and focussing on large presolar SiC grains, i.e., grains larger than $2 \mu \mathrm{m}$ in diameter with mostly moderate recoil effects, Heck et al. (2008, 2009) determined CRE ages ranging from less than 3-1100 Ma; the majority of the grains had CRE in the range of a few tens of Ma up to $200 \mathrm{Ma}$. Using the same data set but improving on the correction for recoil losses, Ott et al. (2009) obtained ages that ranged from zero up to $\sim 850 \mathrm{Ma}$; the majority of the grains had ages less than $\sim 200 \mathrm{Ma}$.

To summarize, CRE age studies of presolar grains are compromised by a number of problems. First, by studying spallogenic neon the excess ${ }^{21} \mathrm{Ne}$ needs to be calculated against the background neon, which is inherited from the helium shell of the AGB star. As the neon composition of the helium shell in AGB stars $\sim 5 \mathrm{Ga}$ ago can be modeled but is somewhat parameter dependent, the CRE ages for presolar grains are also model dependent and should therefore be considered as model ages. Similarly, the helium and lithium concentrations must also be corrected for a trapped component, which is again dependent on the used model. CRE ages determined in this way are thus also model ages. Second, the flux and spectral density of GCRs in the ISM $\sim 5 \mathrm{Ga}$ ago are not known and must be assumed. Third, and most importantly, since presolar grains are small, recoil of cosmogenic nuclides is important and must be corrected for. Here we focus on the last two points. Considering the second problem, we improve the modeling for the production rates and the recoil calculations by using a GCR particle spectra for the current ISM that is fully consistent with recent Voyager data (Stone et al. 2013) and which should therefore be close to or at least very similar to the GCR spectrum in the ISM $\sim 5 \mathrm{Ga}$ ago. Considering the recoil loss calculations, all studies so far corrected for recoil losses in a relatively crude way by either using experimentally determined (constant) recoil ranges from irradiation experiments and/or the recoil losses were calculated using simplified nuclear modeling. For example, Heck et al. (2008, 2009) used a fixed recoil range of $2.5 \mu \mathrm{m}$ for ${ }^{21} \mathrm{Ne}$ in $\mathrm{SiC}$. As mentioned above, Ott et al. (2009) improved the interpretation of the same data by using calculated ${ }^{21} \mathrm{Ne}$ recoil distributions. For modeling, however, they only considered ${ }^{21} \mathrm{Ne}$ production from silicon at a projectile energy of $200 \mathrm{MeV}$ and the recoil ranges for ${ }^{3} \mathrm{He}$ were roughly estimated from measured momentum distributions. In $\mathrm{SiC}$ grains, however, cosmogenic nuclides are produced and recoil is induced by a full spectrum of projectiles. Consequently, the recoil correction itself depends on the particle spectrum in the ISM and-to be fully consistent-the production rates must be calculated using the same GCR spectrum that is used for calculating recoil ranges.

In the next section we present the production rate model for cosmogenic nuclides in presolar grains and we discuss the GCR particle spectrum in the ISM. In Section 3 we discuss the recoil model and in Section 4 we present the results for the production rates and the retention curves for cosmogenic nuclides in presolar $\mathrm{SiC}$ grains. We compare our results with previous models and experimental data in Section 5. Section 6 summarizes the exposure ages for presolar $\mathrm{SiC}$ grains determined applying our approach to experimental data from literature (Heck et al. 2008, 2009; Gyngard et al. 2009a, 2009b). The conclusions are given in Section 7.

\section{COSMOGENIC PRODUCTION RATES}

\subsection{GCR Spectrum}

Presolar grains in the ISM are irradiated by GCR particles and cosmogenic nuclides are produced. The production rates can be calculated if the GCR particle spectrum and the excitation functions for all relevant projectile types are known. For the GCR particle one can use the spectral shape given by Castagnoli \& Lal (1980):

$$
J_{\mathrm{GCR}}\left(E, M, c_{\mathrm{p}}\right)=\frac{c_{\mathrm{p}} E\left(E+2 m_{\mathrm{p}} c^{2}\right)(E+x+M)^{-2.65}}{(E+M)\left(E+2 m_{\mathrm{p}} c^{2}+M\right)} .
$$

The following discussion is focused on GCR protons because (i) they represent the major particle type, (ii) most production rates are dominated by proton-induced reactions, and (iii) the discussion can easily be adapted to other projectile types if the energy is taken in terms of energy per nucleon. In Equation (1) $J_{\mathrm{GCR}}$ is the flux density of protons of energy $E$ as a function of $M$ and $c_{\mathrm{p}}$. The total particle flux is $c_{\mathrm{p}}$, the restmass of the proton is $m_{\mathrm{p}}, c$ is the speed of light, and $x$ is a factor defined as $x=780 \times \exp \left(-2.5 \times E \times 10^{-4}\right)$. The parameter $M$ is the solar modulation parameter of the GCR spectrum, which is essentially the lowest energy a GCR particle must have to travel all the way from the ISM toward the Sun. An often used average for the last few million years is $M=550 \mathrm{MeV}$ (e.g., Leya \& Masarik 2009). The best estimate for the integral number of GCR particles in the asteroid belt in the last few million years is $4.47 \mathrm{~cm}^{-2} \mathrm{~s}^{-1}$ (at $M=550 \mathrm{MeV}$; Leya \& Masarik 2009).

Presolar grains, however, were irradiated by a GCR spectrum that was (most likely) not modulated by stellar magnetic fields and therefore had a different spectral shape and a higher integral particle flux. Recent Voyager data show that the shape of the current GCR spectrum in the ISM, i.e., outside the influence of the solar magnetic field, can be described by Equation (1) when choosing $M=0 \mathrm{MeV}$. While Overholt \& Melott (2013) suggest a very low modulation, the spectral form 
given by Stone et al. (2013) is best described by a modulation $M=0 \mathrm{MeV}$. Integrating the measured GCR spectrum outside the solar system (Stone et al. 2013) gives a total particle flux of $17.3 \mathrm{~cm}^{-2} \mathrm{~s}^{-1}$ and a composition of $93 \%$ protons and $7 \% \alpha$ particles. These data are consistent with the best estimate for the asteroid belt, i.e., for the spectrum within the solar system. For example, a value of $4.47 \mathrm{~cm}^{-2} \mathrm{~s}^{-1}$ for $M=550 \mathrm{MeV}$ corresponds to $\sim 20.8 \mathrm{~cm}^{-2} \mathrm{~s}^{-1}$ for $M=0 \mathrm{MeV}$, i.e., for outside the solar system. The GCR composition within the solar system is $87 \%$ protons, $12 \% \alpha$ particles, and $1 \%$ heavier nuclei (Simpson 1983). We can conclude that the integral flux of the current GCR spectrum outside the solar system (Stone et al. 2013) is in very good agreement with the few million year average of the integral GCR flux inside the solar system.

\subsection{Cosmogenic Production Rates}

All production rate and recoil losses were calculated using the GCR spectrum given by Equation (1) and using $M=0 \mathrm{MeV}$. For modeling we used $J_{\mathrm{p}}=16.1 \mathrm{~cm}^{-2} \mathrm{~s}^{-1}$ $(=17.3 \times 0.93)$ and an $\alpha$ particle fluence of $J_{\alpha}=1.2 \mathrm{~cm}^{-2} \mathrm{~s}^{-1}(=17.3 \times 0.07)$, i.e., consistent with the data given by (Stone et al. 2013). The production rate $P_{j}$ of a cosmogenic nuclide $j$ is calculated via:

$$
P_{j}(M)=\sum_{i=1}^{N} c_{i} \frac{N_{A}}{A_{i}} \sum_{k} \int_{0}^{\infty} \sigma_{j, i, k}(E) J_{k}\left(E, M, c_{k}\right) d E .
$$

Here $c_{i}$ is the concentration for one out of $N$ target elements from which the cosmogenic nuclide $j$ can be produced, $A_{i}$ is the molar mass of target element $i$, and $N_{\mathrm{A}}$ is Avogadro's number. As reaction particle types (denoted by $k$ ) we consider protons and $\alpha$ particles. The excitation function for the production of product $j$ from target element $i$ induced by projectile type $k$ is given by $\sigma_{j, i, k}$. Since presolar grains have typically sizes smaller than $1 \mu \mathrm{m}$ and even the large (atypical) grains we are interested in here have sizes in the range of few tens of micrometers, we assume homogenous production of cosmogenic nuclides in the grain, i.e., stopping and absorption of projectiles is not included in the calculations. In addition, we assume that all secondary particles leave the grain before inducing further nuclear reactions.

Here we report production rates for proton- and $\alpha$-induced reactions. For most of the proton-induced reactions we can rely on experimental data. Note, most of the proton-induced crosssections have been used before to calculate cosmogenic production rates in various types of meteorites and micrometeorites, see, e.g., Ammon et al. (2009), Leya \& Masarik (2009), and Trappitsch \& Leya (2013). The cross-section database for $\alpha$-induced reactions is rather scarce. Here we use the database recently compiled by Trappitsch \& Leya (2013), which has been calculated using the nuclear model codes TALYS-1.2 (Koning et al. 2008) for projectile energies up to $240 \mathrm{MeV}$ and INCL4.5/ABLA07 (Boudard et al. 2002) for higher projectile energies. Here we had to extend the database to the proton-induced production of ${ }^{3} \mathrm{He}$ from ${ }^{\text {nat }} \mathrm{C},{ }^{6,7} \mathrm{Li}$ from ${ }^{\text {nat }} \mathrm{C}$ and ${ }^{\text {nat }} \mathrm{Si}$, and to the $\alpha$-induced production of ${ }^{6,7} \mathrm{Li}$ from ${ }^{\text {nat }} \mathrm{C}$ and ${ }^{\text {nat }} \mathrm{Si}$ (see below).

\subsection{Cross-sections for Proton-induced Reactions}

$$
\text { 2.3.1. }{ }^{\text {nat }} \mathrm{C}(p, X)^{3} \mathrm{He}
$$

We used the TALYS-1.2 code (Koning et al. 2008) to calculate the cross-sections up to an incident proton energy of
$240 \mathrm{MeV}$. In addition we used the experimental data from Kruger \& Heymann (1973) at $600 \mathrm{MeV}$ and $3 \mathrm{GeV}$. The TALYS-1.2 calculation shows two local maxima in the range 19-22 MeV and 46-190 MeV. Since such structures, which typically indicate special evaporation channels, are unphysical and most likely due to computational artifacts these regions were not considered any further. We instead constructed the excitation function by logarithmically interpolating the regions above and below each maximum. In addition, we increased the thus modified TALYS results by a factor of 2.6 to obtain a smooth transition between modeled and measured crosssections (Kruger \& Heymann 1973). Finally, we extended the excitation function to $10 \mathrm{GeV}$ by logarithmic extrapolation; the thus determined cross-section at $10 \mathrm{GeV}$ is $43 \mathrm{mb}$. Note that INCL4.5/ABLA07 (Boudard et al. 2002) is unable to calculate cross-sections for this reaction.

$$
\text { 2.3.2. }{ }^{n a t} C(p, X){ }^{6,7} \mathrm{Li}
$$

We used the TALYS-1.2 code (Koning et al. 2008) only for calculating threshold energies; the shape of the excitation functions were calculated using INCL4.5/ABLA07 (Boudard et al. 2002). In addition, some experimental cross-sections for the production of ${ }^{6,7} \mathrm{Li}$ from ${ }^{12} \mathrm{C}$ exist in the literature (Davids et al. 1970; Raisbeck et al. 1972). We start the discussion with the excitation functions for the production of ${ }^{6,7} \mathrm{Li}$ from ${ }^{12} \mathrm{C}$.

The threshold energies calculated using TALYS-1.2 are for both reactions in good agreement with experimental data (Davids et al. 1970) and can therefore be used for the compilation. In the energy range $28 \mathrm{MeV}-44 \mathrm{MeV}$ we rely on the experimental data (Davids et al. 1970). At higher energies there are measured cross-sections at 150 and $600 \mathrm{MeV}$ (Raisbeck et al. 1972). For the compilation only the crosssection at $150 \mathrm{MeV}$ is used; in the energy range $300 \mathrm{MeV}-$ $10 \mathrm{GeV}$ the excitation function is based on INCL4.5/ABLA07 results. Since the code predicts for both reactions at $600 \mathrm{MeV}$ cross-sections that are lower than the experimental data Raisbeck et al. (1972), we increased the modeled excitation functions for the production of ${ }^{6} \mathrm{Li}$ and ${ }^{7} \mathrm{Li}$ from ${ }^{12} \mathrm{C}$ by factors 4.1 and 1.4 , respectively, for a smooth transition between measured and modeled cross-sections.

No experimental data are available for the production of ${ }^{6,7} \mathrm{Li}$ from ${ }^{13} \mathrm{C}$. The threshold energies were again calculated using TALYS-1.2 and the shape of the excitation functions were calculated using INCL4.5/ABLA07. To be consistent with the production of ${ }^{6,7} \mathrm{Li}$ from ${ }^{12} \mathrm{C}$ (see above), we also increased the INCL4.5/ABLA07 results for the production of ${ }^{6} \mathrm{Li}$ and ${ }^{7} \mathrm{Li}$ from ${ }^{13} \mathrm{C}$ by factors 4.1 and 1.4 , respectively. The excitation functions for the production of ${ }^{6,7} \mathrm{Li}$ from ${ }^{\text {nat }} \mathrm{C}$ were calculated assuming solar composition of carbon, i.e., $98.93 \%{ }^{12} \mathrm{C}$ and $1.07 \%{ }^{13} \mathrm{C}$. Figure 1 (top panel) shows the compiled excitation functions together with experimental data.

$$
\text { 2.3.3. }{ }^{n a t} \mathrm{Si}(p, X)^{6,7} \mathrm{Li}
$$

We used INCL4.5/ABLA07 (Boudard et al. 2002) to calculate the excitation functions for the production of ${ }^{6,7} \mathrm{Li}$ from ${ }^{\text {nat }} \mathrm{Si}$. TALYS-1.2 could not be used because it cannot be applied for large mass differences between target and products. Since INCL4.5/ABLA07 is not always reliable for the production of very light product nuclides, we tested the predictions on the very similar reaction ${ }^{n a t} \mathrm{Si}(\mathrm{p}, \mathrm{X}){ }^{7} \mathrm{Be}$. Based on the experimental data at $1.2 \mathrm{GeV}$ (Michel et al. 1995) and 


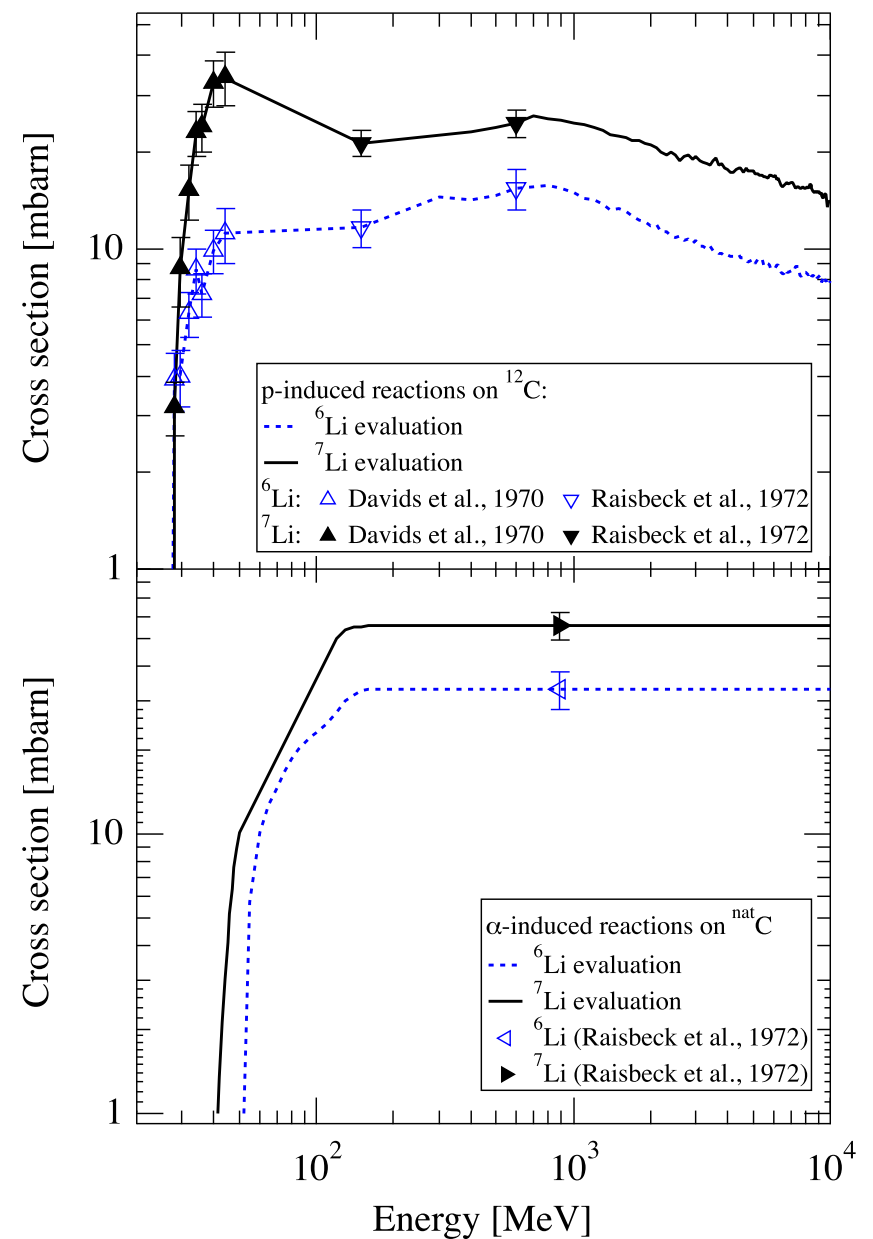

Figure 1. Evaluated excitation functions for the reactions ${ }^{\text {nat }} \mathrm{C}(\mathrm{p}, \mathrm{X}){ }^{6,7} \mathrm{Li}$ (top panel) and ${ }^{\text {nat }} \mathrm{C}(\alpha, \mathrm{X}){ }^{6,7} \mathrm{Li}$. Also shown are experimental data. For more information see text.

1.6 GeV (Michel et al. 1995; Leya 1997) it can be concluded that the model predictions must be downscaled by an average factor of 1.5 to match the data. Because of the similar reaction mechanism we adjusted the modeled cross-sections for the production of ${ }^{6,7} \mathrm{Li}$ from ${ }^{\text {nat }} \mathrm{Si}$ by the same factor of 1.5 .

\subsection{Cross-sections for $\alpha$-induced Reactions}

In contrast to the proton-induced reactions, the database for $\alpha$-induced reactions is still very scarce, which limits the possibilities to check the quality of the calculated crosssections. For the production of helium and neon from carbon and silicon we used the same approach as Trappitsch \& Leya (2013), i.e., we calculated cross-sections up to $240 \mathrm{MeV}$ using the TALYS-1.2 code and assumed constant cross-sections up to $10 \mathrm{GeV}$.

$$
\text { 2.4.1. }{ }^{\text {nat }} C(\alpha, X){ }^{6,7} \mathrm{Li}
$$

As mentioned above, INCL4.5/ABLA07 cannot be used to calculate excitation functions for $\mathrm{p}$ - or $\alpha$-induced reactions on ${ }^{12} \mathrm{C}$. We therefore used TALYS-1.2 to calculate cross-sections up to $240 \mathrm{MeV}$. However, because the calculated data drop sharply at $E>170 \mathrm{MeV}$, which we consider as a numerical artifact, those data were not considered any further. In addition, the calculated excitation function for the production of ${ }^{7} \mathrm{Li}$ shows a local maximum in the range $55-110 \mathrm{MeV}$, which is not expected from nuclear reaction systematics and which we therefore replaced by a logarithmic interpolation between areas at lower and higher energies. The thus modeled excitation functions were then extended with experimental data at $880 \mathrm{MeV}$ (Raisbeck et al. 1972) and assuming constant crosssections in the range $880 \mathrm{MeV}-10 \mathrm{GeV}$ (bottom panel in Figure 1) using a simple double logarithmic interpolation.

$$
\text { 2.4.2. }{ }^{n a t} \operatorname{Si}(\alpha, X){ }^{6,7} \mathrm{Li}
$$

As mentioned above, TALYS-1.2 cannot be applied to reactions with large target-product-mass differences. In addition, INCL4.5/ABLA07 predictions for $\alpha$-induced reactions are unreliable. In order to be nevertheless able to estimate production rates, we compared the rates for the $\mathrm{p}$ - and $\alpha$ induced production of ${ }^{6,7} \mathrm{Li}$ from ${ }^{\text {nat }} \mathrm{C}$. The ratios of $\alpha$ - to proton-induced production for ${ }^{6} \mathrm{Li}$ from ${ }^{\text {nat }} \mathrm{C}$ is 2.56 , for ${ }^{7} \mathrm{Li}$ it is 2.44. Assuming the same $\alpha$ - to proton-induced production ratios also for reactions on ${ }^{\text {nat }} \mathrm{Si}$, we divided the $\mathrm{p}$-induced production rates for ${ }^{6} \mathrm{Li}$ and ${ }^{7} \mathrm{Li}$ from ${ }^{\text {nat }} \mathrm{Si}$ by factors 2.56 and 2.44 , respectively.

\subsection{Uncertainties of Cross-sections}

The excitation functions for the proton-induced production of helium and neon isotopes from silicon are mostly based on measured data that typically have uncertainties in the range $5 \%-10 \%$. Any results from nuclear modeling were adjusted to experimental data; we therefore also assign uncertainties of $10 \%$ to the calculated values. For the production of ${ }^{6,7} \mathrm{Li}$ fom ${ }^{n a t} \mathrm{C}$ there are less experimental data and we therefore assigned an uncertainty of $20 \%$ to the excitation functions. For the reactions ${ }^{\text {nat }} \mathrm{C}(\mathrm{p}, \mathrm{X}){ }^{3} \mathrm{He}$ and ${ }^{\text {nat }} \mathrm{C}(\mathrm{p}, \mathrm{X}){ }^{6,7} \mathrm{Li}$ we assume that the uncertainties are in the range $50 \%$ because even fewer experimental data are available and we have to rely more on nuclear modeling. For the production of ${ }^{6,7} \mathrm{Li}$ from ${ }^{\text {nat }} \mathrm{Si}$ no experimental cross-sections are available and we had to significantly scale the excitation function; we therefore assign an uncertainty of a factor of two to these excitation functions.

The database for $\alpha$-induced reactions is worse; for most relevant reactions no experimental data are available and we have to rely on calculated cross-sections. For the production of ${ }^{6,7} \mathrm{Li}$, for which few experimental data are available, we assign an uncertainty of $50 \%$ to the excitation functions. For all other $\alpha$-induced reactions, for which no experimental data are available, we assign an uncertainty of a factor of four to the excitation function, which is the maximum scaling factor used for our compilation (see above). Note that the uncertainties of the excitation functions directly propagate into the uncertainties for the modeled production rates.

\section{RECOIL LOSS}

The model used here is essentially the same as in Trappitsch \& Leya (2013). However, the former model was for technical reasons limited to protons with energies lower than $240 \mathrm{MeV}$, which was not a limiting factor for cosmogenic production rates in micrometeorites but is a serious limitation for cosmogenic production rates in presolar grains. Below we briefly describe how the the model is extended to higher energies (up to $10 \mathrm{GeV}$ ) and to $\alpha$-particles as projectiles.

Up to an incident proton energy of $240 \mathrm{MeV}$ the production cross-sections and recoil spectra for all relevant target-product combinations were calculated using TALYS-1.2 (Koning 


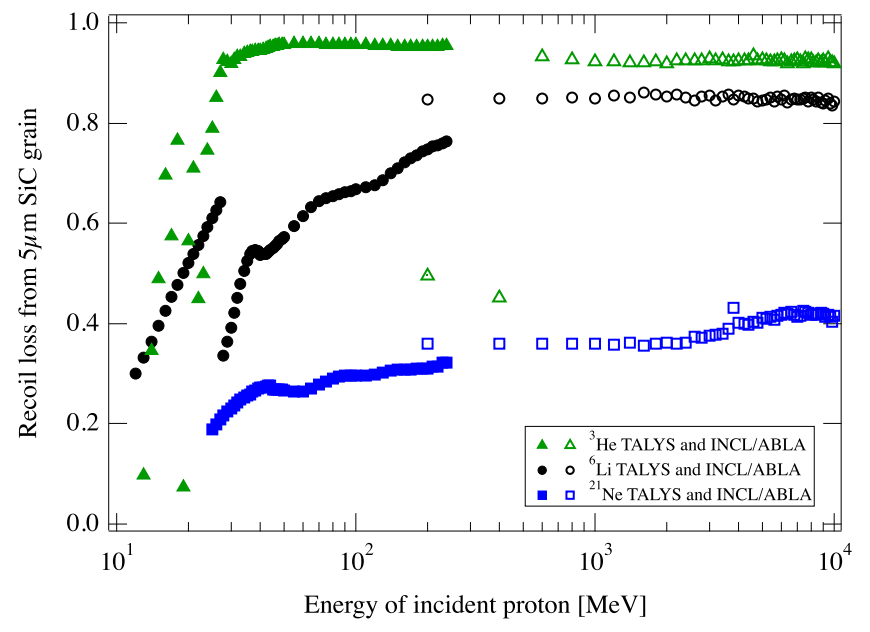

Figure 2. Recoil losses of ${ }^{3} \mathrm{He},{ }^{6} \mathrm{Li}$, and ${ }^{21} \mathrm{Ne}$ for a $5 \mu \mathrm{m} \mathrm{SiC}$ grain as a function of incident proton energy. Below $200 \mathrm{MeV}$ the results are based on TALYS1.2 predictions (solid symbols). At higher energies the recoil losses are based on INCL4.5/ABLA07 results (open symbols).

et al. 2008). To increase the energy resolution, we interpolated the recoil spectra linearly to a resolution of $0.1 \mathrm{MeV}$. In addition, we linearly extrapolated the recoil spectra toward zero recoil energy, which is important for very small grains because it accounts for the fact that some product nuclides have a recoil energy of zero and can therefore remain even in the smallest grains. For each target-product combination, incident proton energy, and recoil energy we calculated the stopping range using the PRAL algorithm (Biersack 1981). The electronic and nuclear stopping parameters were calculated using the srmodule software of the SRIM package (Ziegler 2004). Depending on the stopping range, the geometry of the target grain, the production location within the grain, and the momentum vector of the produced cosmogenic nuclide, we calculated whether or not the product nuclide is lost from the grain using the algorithm described in detail by Trappitsch \& Leya (2013).

Since being consistent is very important for this type of calculations, we entirely rely on data determined using nuclear model codes. We expect that both models, i.e., TALYS-1.2 and INCL4.5/ABLA, can reliably predict relative values, i.e., cross-sections and recoil spectra of one isotope relative to another isotope. This is confirmed by the finding that TALYS1.2 calculates solar cosmic ray (SCR) produced ${ }^{21} \mathrm{Ne} /{ }^{22} \mathrm{Ne}$ ratios, i.e., relative production rates, very accurate (Meier et al. 2014). In addition, Trappitsch \& Leya (2013) used the very same model to calculate recoil losses for the production of ${ }^{21} \mathrm{Ne}$ from silicon at $200 \mathrm{MeV}$ incident proton energy and found that the calculations describe existing experimental values (Ott \& Begemann 2000) very well.

\subsection{Recoil Spectra for Proton-induced Reactions}

For incident energies up to $240 \mathrm{MeV}$ we use the model given by Trappitsch \& Leya (2013; see above). At higher energies the cross-sections and recoil spectra were calculated using INCL4.5/ABLA07 (Boudard et al. 2002), which is a Monte Carlo code for the simulation of nuclear reactions in the energy range $200 \mathrm{MeV}-10 \mathrm{GeV}$. The INCL4.5/ABLA07 results are formatted to match the TALYS format. Figure 2 depicts relative recoil losses for the proton-induced production of ${ }^{3} \mathrm{He}$, ${ }^{6} \mathrm{Li}$, and ${ }^{21} \mathrm{Ne}$ in a $5 \mu \mathrm{m} \mathrm{SiC}$ grain as a function of incident proton energy. In this plot a value of one indicates complete loss. Note that the data have been calculated using TALYS-1.2 below $240 \mathrm{MeV}$ (solid symbols) and using INCL4.5/ABLA07 in the energy range $200 \mathrm{MeV}-10 \mathrm{GeV}$ (open symbols). For all three nuclides there is a perfect match between TALYS-1.2 and INCL4.5/ABLA07 results. For example, the recoil ranges in the overlapping energy range at $200 \mathrm{MeV}$ differ by less than $10 \%$ and $5 \%$ for ${ }^{6} \mathrm{Li}$ and ${ }^{21} \mathrm{Ne}$, respectively. For ${ }^{3} \mathrm{He}$ the differences between both approaches are slightly larger. However, ignoring the first two datapoints calculated using INCL4.5/ABLA07 at $200 \mathrm{MeV}$ and $400 \mathrm{MeV}$ the agreement for both nuclear model codes is excellent, i.e., the difference is in the range of only $2 \%$.

Figure 2 shows that the recoil losses for ${ }^{3} \mathrm{He}$ and ${ }^{6} \mathrm{Li}$ scatter widely close to the reaction threshold, which we consider computational artifacts caused by low cross-sections. However, these artifacts have only little effect on the calculated recoil losses, which has been checked by calculating ${ }^{3} \mathrm{He}$ and ${ }^{6} \mathrm{Li}$ recoil losses from a $5 \mu \mathrm{m} \mathrm{SiC}$ grain using the entire GCR spectrum. The first set of modeling fully considers the widely varying low-energy part of the recoil losses and in the second set of modeling we artificially set all recoil losses below $22 \mathrm{MeV}$ for ${ }^{3} \mathrm{He}$ and below $28 \mathrm{MeV}$ for ${ }^{6} \mathrm{Li}$ to zero. The differences between both modeling types are $2 \%$ for ${ }^{3} \mathrm{He}$ and $<0.1 \%$ for ${ }^{6} \mathrm{Li}$, clearly demonstrating that the scatter in the low-energy data has only little influence on the final results.

\section{2. $\alpha$-induced Reactions}

As already mentioned, INCL4.5/ABLA07 fails or has difficulties modeling $\alpha$-induced reactions on carbon and silicon. We were therefore unable to calculate recoil losses for $\alpha$-induced reactions at high projectile energies. To overcome this problem we assume that the recoil losses for $\alpha$ induced reactions are constant above $240 \mathrm{MeV}$. Though, this assumption adds some uncertainties to the modeling, it is justified by the fact that recoil losses for proton-induced reactions are also constant at high energies. Neither TALYS1.2 nor INCL4.5/ABLA07 can model the production of and/or the recoil losses for ${ }^{6,7} \mathrm{Li}$ from ${ }^{\text {nat }} \mathrm{Si}$. To overcome this limitation we assume that the recoil losses are the same as for the production of ${ }^{6,7} \mathrm{Li}$ by $\alpha$-induced reactions on carbon. Though, this will add some uncertainties, we expect them to be only very minor because $\alpha$-particles contribute only $7 \%$ to the GCR. Figure 3 compares recoil spectra for the proton- and $\alpha$ induced production of ${ }^{21} \mathrm{Ne}$ from ${ }^{\text {nat }} \mathrm{Si}$ at $40 \mathrm{MeV}$ and $240 \mathrm{MeV}$. The general trend is similar for both projectile types; increasing cross-sections per energy at very low recoil energies, local maxima in the range of few $\mathrm{MeV}$, and decreasing values at higher energies. With increasing projectile energy the distribution becomes broader.

\section{RESULTS}

\subsection{Cosmogenic Production Rates}

Table 1 compiles the $\alpha$ - and proton-induced production rates for ${ }^{3} \mathrm{He}$ and ${ }^{6,7} \mathrm{Li}$ from carbon and ${ }^{3} \mathrm{He},{ }^{6,7} \mathrm{Li}$, and ${ }^{21,22} \mathrm{Ne}$ from silicon. The production rates are normalized to proton- and $\alpha$ particle fluxes of $1 \mathrm{~cm}^{-2} \mathrm{~s}^{-1}$ and they are for a GCR spectrum of $M=0 \mathrm{MeV}$. The uncertainties cover only the uncertainties of the excitation functions, uncertainties due to ill-known particle spectra are not included. There is an additional source of uncertainties for the ${ }^{3} \mathrm{He}$ production rates caused by ill- 


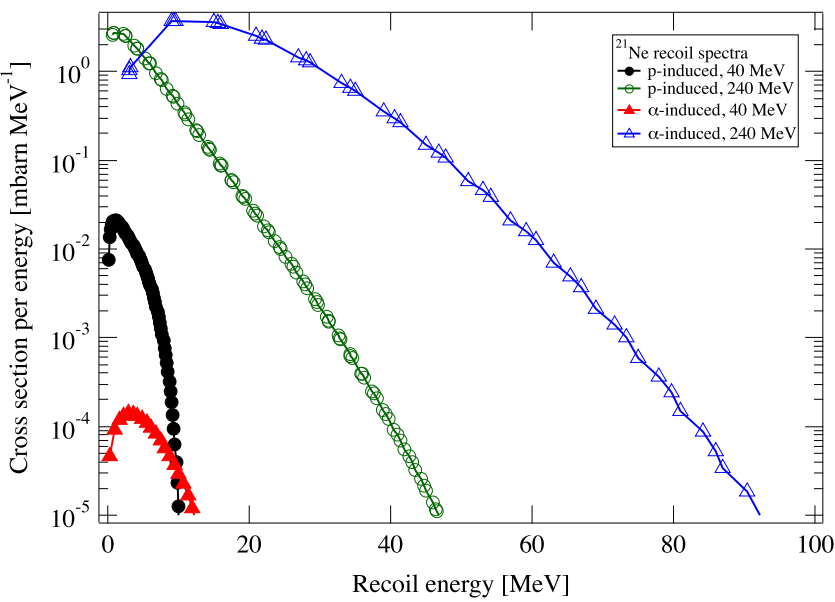

Figure 3. Comparison of recoil spectra for the production of ${ }^{21} \mathrm{Ne}$ from silicon at 40 and $240 \mathrm{MeV}$ (calculated using TALYS-1.2).

known contributions from the radioactive progenitor ${ }^{3} \mathrm{H}$. Since ${ }^{3} \mathrm{H}$ has large diffusion rates, it is relatively safe to assume that in presolar grains all ${ }^{3} \mathrm{H}$ is lost to space before radioactive decay to ${ }^{3} \mathrm{He}$. The production rates given in Table 1 are therefore only for the direct production of ${ }^{3} \mathrm{He}$. If we would as the other extreme assume that all ${ }^{3} \mathrm{H}$ decays to ${ }^{3} \mathrm{He}$ in the presolar grain, i.e., there are no diffusive losses of ${ }^{3} \mathrm{H}$, the ${ }^{3} \mathrm{He}$ production rates from silicon and carbon would increase by factors of about 2.16 and about 1.76, respectively (Leya et al. 2004). The latter value is calculated based on the systematics given by Leya et al. (2004). We estimate that these factors have uncertainties of about $20 \%$, which considers the fact that the ${ }^{3} \mathrm{H} /{ }^{3} \mathrm{He}$ production rate ratio might depend on the shape of the particle spectrum.

\subsection{Recoil Loss}

Figure 4 depicts retention fractions for ${ }^{3} \mathrm{He},{ }^{6,7} \mathrm{Li}$, and ${ }^{21,22} \mathrm{Ne}$ in $\mathrm{SiC}$ grains with radii between 1 and $100 \mu \mathrm{m}$. The retention fractions for the $\alpha$ - and proton-induced production are shown separately in the upper and lower panel, respectively. For the product nuclides studied here retention fractions at the same energy are slightly higher for the proton- than for the $\alpha$ induced production. With the proton abundance, $a_{\mathrm{p}}$, and the $\alpha$ abundance, $a_{\alpha}$, and by considering the production rates, the total recoil loss fraction can be calculated via:

$$
r_{\mathrm{tot}}=\frac{a_{\mathrm{p}} P_{\mathrm{p}} r_{\mathrm{p}}+a_{\alpha} P_{\alpha} r_{\alpha}}{a_{\mathrm{p}} P_{\mathrm{p}}+a_{\alpha} P_{\alpha}}
$$

with $P_{\mathrm{p}}$ and $P_{\alpha}$ the calculated production rates and $r_{\mathrm{p}}$ and $r_{\alpha}$ the recoil losses for the proton- and $\alpha$-induced production, respectively. Figure 5 shows the recoil losses calculated using Equation (3) and assuming that the GCRs consist of $92 \%$ protons and $8 \% \alpha$ particles.

Estimating the uncertainties of the recoil losses is difficult because for modeling we entirely rely on calculated data that cannot be verified using experimental values. We assume rather conservatively an uncertainty of about $50 \%$ for the calculated recoil losses (see below). For real applications, however, the major source of uncertainty is probably not in the modeled data but in the assumed shape of the presolar grains. Here we assume a perfect spherical shape for the grains, which is most likely not the case. Any deviation from the spherical shape, especially considering conglomerates of $\mathrm{SiC}$ grains, increases the recoil losses compared to the modeling. Strictly speaking, the recoil losses calculated here should be considered as lower limits.

\section{COMPARING THE MODEL PREDICTIONS TO LITERATURE VALUES}

\subsection{Cosmogenic Production Rates}

The only pervious study on cosmogenic production rates in presolar grains by Reedy (1989) is based on a GCR particle spectrum with a solar modulation parameter $M=100 \mathrm{MeV}$ and a GCR particle fluence identical to the current value inside the solar system. In contrast, our model uses a GCR spectrum with no modulation and flux densities consistent with values measured by Voyager outside the solar system. A direct comparison is therefore not possible. Just for comparison, using a GCR spectrum with $M=0 \mathrm{MeV}$ but using the (wrong) particle fluence from Reedy (1989) we calculate production rates for ${ }^{3} \mathrm{He}$ about $20 \%$ lower, for ${ }^{6} \mathrm{Li}$ about $60 \%$ higher, for ${ }^{7} \mathrm{Li}$ about $10 \%$ higher, for ${ }^{21} \mathrm{Ne}$ about $10 \%$ higher and for ${ }^{22} \mathrm{Ne}$ about $20 \%$ lower than the production rates by Reedy (1989). The agreement is reasonable and the finding that our production rates for ${ }^{6,7} \mathrm{Li}$ are higher is at least partly due to fact that our model also considers production from silicon, a reaction path not considered by Reedy (1989). The remaining (minor) differences are most likely due to the updated crosssection database used in our model and a more reliable description of the GCR particle spectrum. We therefore consider the new production rates as superior.

For calculating cosmogenic exposure ages using lithium isotopes, the spallogenic ${ }^{6} \mathrm{Li} /{ }^{7} \mathrm{Li}$ ratio has to be known. Figure 6 shows recoil corrected ${ }^{6} \mathrm{Li} /{ }^{7} \mathrm{Li}$ ratios as a function of grain size for presolar $\mathrm{SiC}$.

\subsection{Recoil Model}

Validating the results of the recoil model is difficult because only few experimental data exist. In the energy range 3-300 GeV Steinberg \& Winsberg (1974) studied protoninduced recoil of ${ }^{22} \mathrm{Na}$ and ${ }^{24} \mathrm{Na}$ in aluminum foils with thicknesses $<7 \mu \mathrm{m}$ and found losses in the range of $20 \%$. Using our approach and assuming isotropic recoil momenta for the produced nuclides we calculate values in the range of $20 \%-$ $30 \%$ for energies between $240 \mathrm{MeV}$ and $10 \mathrm{GeV}$. The minor difference between modeled and measured data is most likely due to the relatively crude assumption of isotropic recoil momenta. In addition, Beyer \& Herrmann (1978) and LagardeSimonoff et al. (1976) experimentally showed that above a projectile energy of $200 \mathrm{MeV}$ the energy has little influence on the kinetic energy of the recoil product, hence the recoil loss becomes almost constant. Our model reproduces this behavior very nicely as can be seen in Figure 2.

Previous recoil modeling has been done by Ott \& Begemann (2000) and Ott et al. (2005). In their early study, Ott \& Begemann (2000) assumed a constant recoil range for ${ }^{21} \mathrm{Ne}$. Assuming for our approach the same constant recoil range we are able reproducing their retention curve. As the next step we used our model to reproduce the average recoil range used by these authors. Doing so, we averaged the modeled recoil spectra by weighting all energies with their individual crosssection per energy, which then gives the most likely recoil energy. Using these average energies we then calculated 
Table 1

Elemental Production Rates for Cosmogenic ${ }^{3} \mathrm{He},{ }^{6} \mathrm{Li},{ }^{7} \mathrm{Li},{ }^{21} \mathrm{Ne}$, and ${ }^{22} \mathrm{Ne}$

\begin{tabular}{lcccc}
\hline \hline Cosmogenic Nuclide & \multicolumn{4}{c}{ Elemental Production Rates $\left(10^{-10} \mathrm{~cm}^{3} \mathrm{STP}^{-1} \mathrm{Ma}^{-1}\right)$} \\
\cline { 2 - 5 } & $\mathrm{C}(\mathrm{p})$ & $\mathrm{C}(\alpha)$ & $\mathrm{Si}(\mathrm{p})$ & \\
\hline${ }^{3} \mathrm{He}$ & $21.8 \pm 2.18$ & $48.7 \pm 24.3$ & $9.83 \pm 4.92$ & \\
${ }^{6} \mathrm{Li}$ & $7.44 \pm 1.49$ & $19.0 \pm 9.52$ & $9.66 \pm 9.66$ & $24.3 \pm 201$ \\
${ }^{7} \mathrm{Li}$ & $13.2 \pm 2.65$ & $32.3 \pm 16.2$ & $8.12 \pm 8.12$ & $19.85 \pm 79.39$ \\
${ }^{21} \mathrm{Ne}$ & $\cdots$ & $\cdots$ & $4.65 \pm 0.465$ & $5.44 \pm 21.8$ \\
${ }^{22} \mathrm{Ne}$ & $\cdots$ & $\cdots$ & $5.79 \pm 0.579$ & $6.29 \pm 25.2$ \\
\hline
\end{tabular}

Note. Calculations were done assuming either protons or $\alpha$ particles as projectiles, a GCR spectrum with a modulation of $0 \mathrm{MeV}$ and a flux density of $1 \mathrm{~cm}^{-2} \mathrm{~s}^{-1}$. The given uncertainties are solely from the cross-section uncertainties (see text).

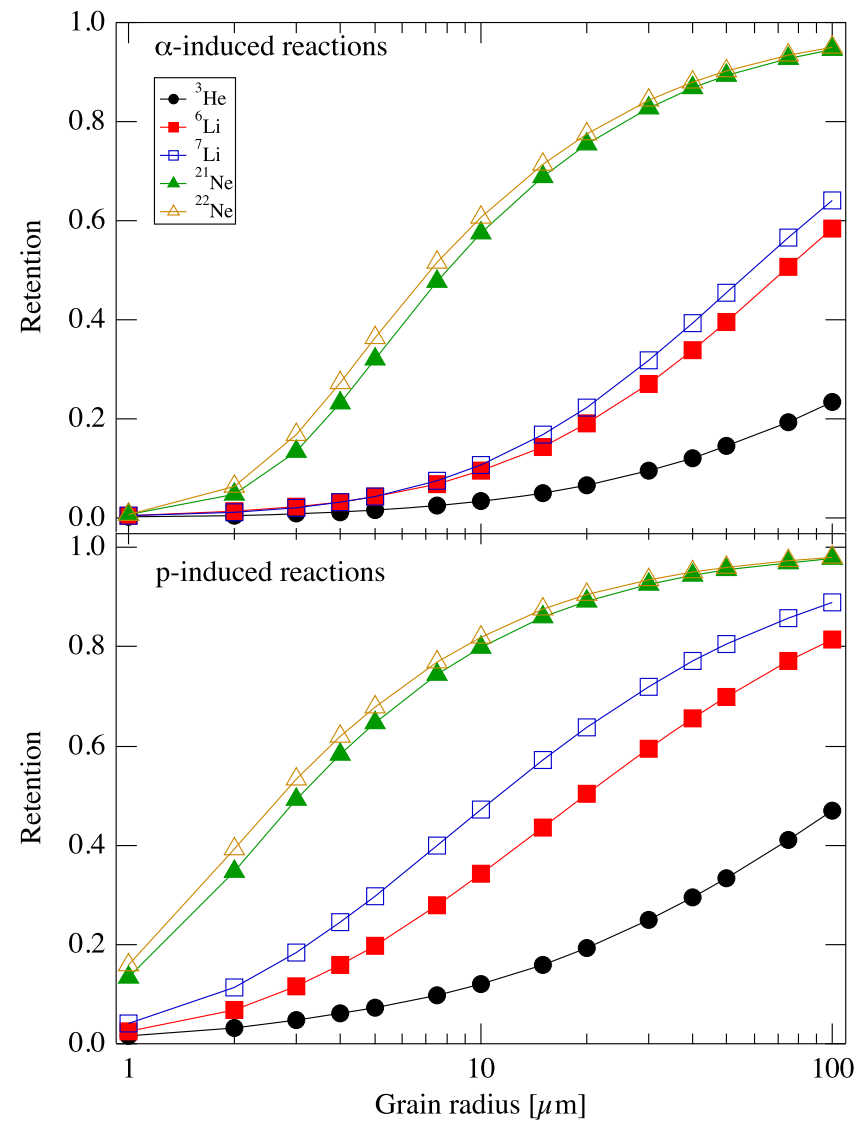

Figure 4. Retention of cosmogenic nuclides in $\mathrm{SiC}$ grains as a function of grain size. The top panel shows the retention for $\alpha$-induced production; the lower panel shows the retention for the proton-induced production.

average recoil ranges for each isotope. Based on experimental data for ${ }^{21} \mathrm{Ne}$ recoil at $1.6 \mathrm{GeV}$, Ott \& Begemann (2000) calculated a recoil range for ${ }^{21} \mathrm{Ne}$ in $\mathrm{SiC}$ of $2.5 \mu \mathrm{m}$. Later, Ott et al. (2005) measured recoil ranges of $2 \mu \mathrm{m}$ for the protoninduced production of ${ }^{22} \mathrm{Na}$ from silicon and aluminum at $66 \mathrm{MeV}$. Using the model we calculate recoil ranges for the production of ${ }^{22} \mathrm{Na}$ from silicon and aluminum of $2.51 \mu \mathrm{m}$ and $2.39 \mu \mathrm{m}$, respectively. For the target element barium Ott et al. (2005) measured recoil ranges for ${ }^{127} \mathrm{Xe}$ at $66 \mathrm{MeV}$ of $<1.7 \mu \mathrm{m}$ and for ${ }^{126} \mathrm{Xe}$ at $267 \mathrm{MeV}$ of $0.21 \mu \mathrm{m}$. The modeled recoil range for ${ }^{127} \mathrm{Xe}$ at $66 \mathrm{MeV}$ is $0.32 \mu \mathrm{m}$ and for ${ }^{126} \mathrm{Xe}$ at $240 \mathrm{MeV}$ it is $0.47 \mu \mathrm{m}$, i.e., both values are reasonably close to the measured data. At a proton energy of $1.2 \mathrm{GeV}$, Ott et al. (2005) reported a recoil range for ${ }^{126} \mathrm{Xe}$ produced from barium of $0.16 \mu \mathrm{m}$. Unfortunately, our model does not include

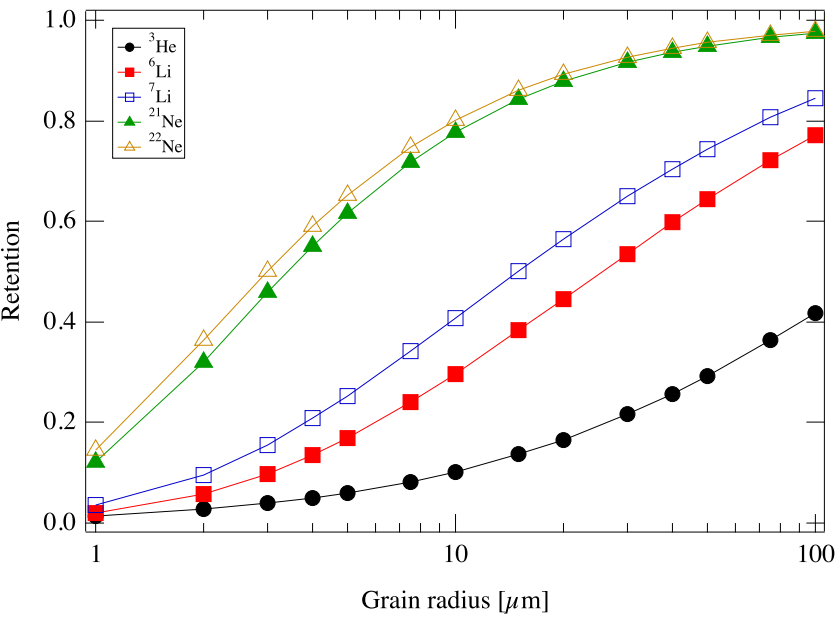

Figure 5. Retention of cosmogenic nuclides in $\mathrm{SiC}$ grains as a function of grain size for a GCR spectrum of $93 \%$ protons and $7 \% \alpha$-particles. For more information see text.

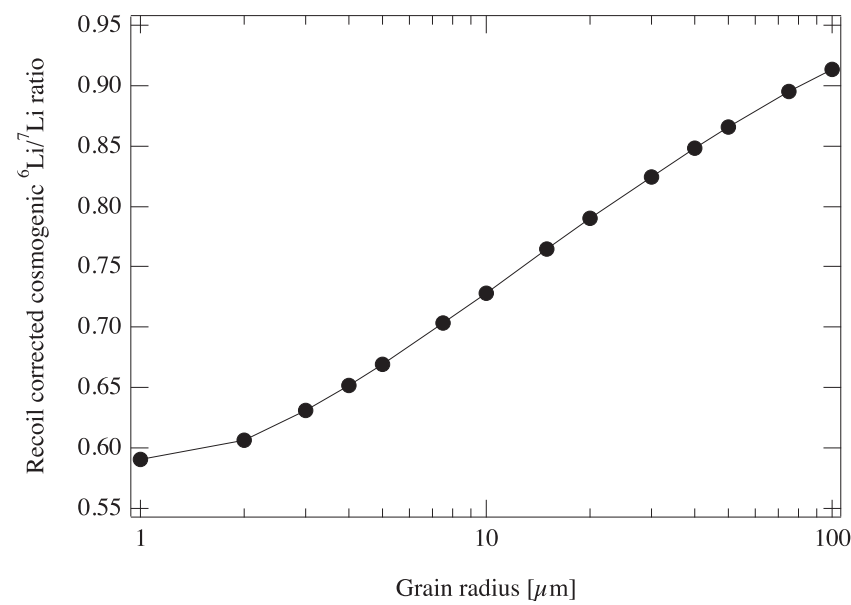

Figure 6. Recoil loss corrected cosmogenic ${ }^{6} \mathrm{Li} /{ }^{7} \mathrm{Li}$ ratio as a function of grain size for presolar $\mathrm{SiC}$ grains.

INCL4.5/ABLA07 predictions for the target element barium, making a direct comparison impossible. However, considering that recoil energies are almost constant at higher projectile energies (see above), the measured value at $1.2 \mathrm{GeV}$ (Ott et al. 2005) agrees reasonably well with our modeled value at $240 \mathrm{MeV}$.

There are three points that needs to be emphasized. First, Ott \& Begemann (2000) explored the possibility that the produced cosmogenic nuclides have no recoil energy; a fact that could not be quantified in their approach of constant recoil ranges but 
Table 2

Production Rates of Cosmogenic Nuclides in SiC Grains using the Elemental Production Rates from Table 1, assuming a GCR Composition of 93\% Protons and $7 \% \alpha$ Particles, and an Integral Particle Flux of $17.3 \mathrm{~cm}^{-2} \mathrm{~s}^{-1}$ (Stone et al. 2013)

\begin{tabular}{lc}
\hline \hline Cosmogenic & $\begin{array}{c}\text { Production Rate } \\
\text { Nuclide }\end{array}$ \\
\hline${ }^{3} \mathrm{He}$ & $317 \pm 135$ \\
${ }^{6} \mathrm{Li}$ & $165 \pm 101$ \\
${ }^{7} \mathrm{Li}$ & $205 \pm 85.7$ \\
${ }^{21} \mathrm{Ne}$ & $40.8 \pm 14.3$ \\
${ }^{22} \mathrm{Ne}$ & $50.6 \pm 16.6$ \\
\hline
\end{tabular}

that is a natural outcome in our model and that is needed explaining why there is no complete recoil loss even in very small grains. Second, Lagarde-Simonoff et al. (1976) demonstrated that the recoil range of a given nuclide at a given incident proton energy is linearly correlated via $d A / A$, with $A$ the mass of the target nuclide and $d A$ the mass difference between target and product. Ott et al. (2005) confirmed this linear relationship for barium isotopes. The same type of linear correlation can also be reproduced using our model. Note that Lagarde-Simonoff et al. (1976) analyzed recoil products that are heavier than the ones studied here. It is therefore difficult to directly compare their results to ours but it is worth mentioning that according to the model, the light recoil corrections we are interested in follow the same type of correlation. Third, the recoil calculations by Gyngard et al. (2009a) for lithium isotopes are based on data for lithium production from carbon and oxygen (Greiner et al. 1975). However, their recoil spectrum has the maximum at much lower energies than we determined using the more reliable TALYS-1.2 and/or INCL4.5/ABLA07 codes. In addition, the data by Greiner et al. (1975) yield a poor agreement with the linear correlation given by Lagarde-Simonoff et al. (1976) that is confirmed by our model. Using the linear correlation we expect that the recoil ranges used by Gyngard et al. (2009a) are too small, which is indeed what we confirmed using the model calculations (see above).

\section{CRE AGES OF PRESOLAR GRAINS}

We calculated cosmogenic production rates in presolar $\mathrm{SiC}$ grains using a GCR particle fluence in the ISM of $17.3 \mathrm{~cm}^{-2} \mathrm{~s}^{-1}$, a solar modulation parameter $M=0 \mathrm{MeV}$, and a GCR composition of $93 \%$ protons and $7 \% \alpha$ particles (Table 2, see also above). Using the new production rates and considering recoil losses we reevaluated the data published by Gyngard et al. (2009a, 2009b) and Heck et al. (2008, 2009) to determine improved CRE ages for the first time fully considering the uncertainties of the production rates. Note that all of these measurements were performed on a selected subset of presolar $\mathrm{SiC}$ grains, i.e., large grains that are not necessarily representative of the majority of the presolar grain collection. However, small grains are more difficult to study due to their much lower abundance of cosmogenic nuclides and due to the fact that recoil corrections in small grains are large and are thus more uncertain. For example, even cosmogenic neon isotopes, which have the lowest recoil losses of the studied cosmogenic nuclides, have a retention fraction of less than $20 \%$ in grains smaller than $1 \mu \mathrm{m}$ radius.

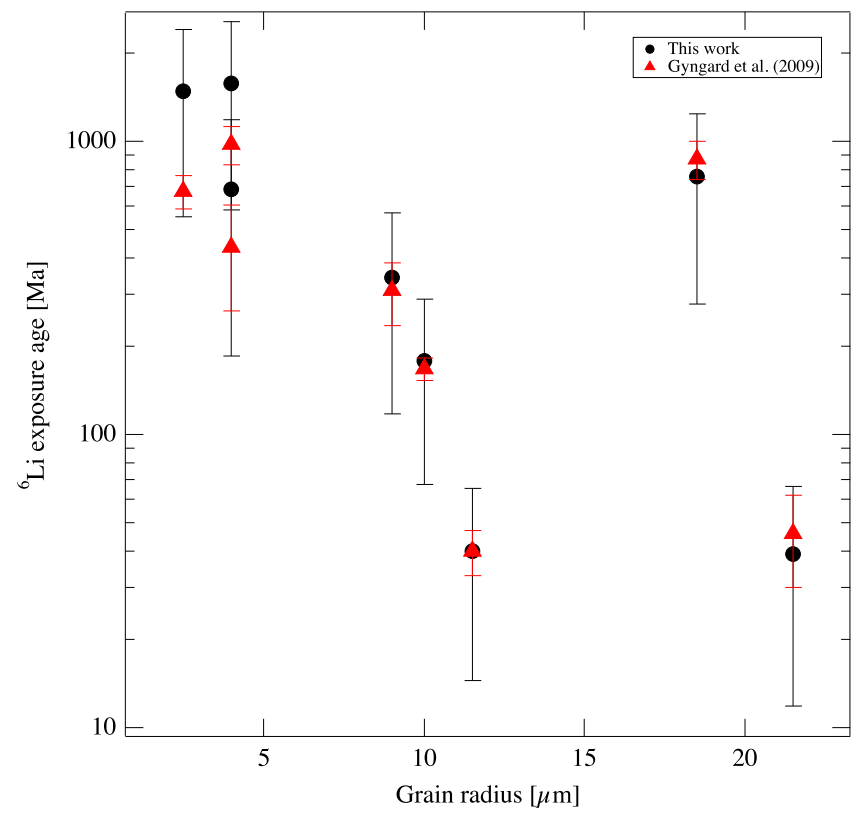

Figure 7. CRE ages based on the lithium isotope data measured by Gyngard et al. (2009a, 2009b) as a function of grain size. The original ages are shown by red triangles; the reevaluated ages are shown by black dots.

Table 3

Reevaluated Retention Fractions and Grain Ages for the Presolar SiC Grains Measured by Gyngard et al. (2009a)

\begin{tabular}{lcccc}
\hline \hline $\begin{array}{c}\text { Grain } \\
\text { Label }\end{array}$ & $\begin{array}{c}\text { Grain Radius } \\
(\mu \mathrm{m})\end{array}$ & $\begin{array}{c}\text { Retention } \\
\text { of }{ }^{6} \mathrm{Li}\end{array}$ & $\begin{array}{c}\text { Retention } \\
\text { of }{ }^{7} \mathrm{Li}\end{array}$ & $\begin{array}{c}T_{6} \\
(\mathrm{Ma})\end{array}$ \\
\hline $\mathrm{a} 3-3$ & 18.5 & 0.429 & 0.548 & $757 \pm 478$ \\
$\mathrm{a} 4-2$ & 4.00 & 0.136 & 0.208 & $1572 \pm 991$ \\
$\mathrm{a} 4-4$ & 11.5 & 0.326 & 0.440 & $40 \pm 26$ \\
a4-5 & 21.5 & 0.462 & 0.580 & $39 \pm 27$ \\
a5-1 & 2.50 & 0.078 & 0.127 & $1487 \pm 931$ \\
b3-2 & 9.00 & 0.276 & 0.384 & $344 \pm 226$ \\
b3-1 & 10.0 & 0.297 & 0.408 & $178 \pm 110$ \\
b3-4 & 4.00 & 0.136 & 0.208 & $687 \pm 501$ \\
\hline
\end{tabular}

Note. Grain labels and sizes are taken from Gyngard et al. (2009a).

\subsection{CRE Ages Based on Lithium Isotope Data}

The CRE ages determined from the lithium data by Gyngard et al. (2009a, 2009b) are shown in Figure 7. The newly calculated CRE ages and retention fractions are given in Table 3. Unfortunately, Gyngard et al. (2009a, 2009b) did not report uncertainties for the ages. The error bars shown in Figure 7 only include the reported uncertainties for the measurements, they do not include any uncertainties from the production rates and the recoil correction. In contrast, the uncertainties shown for the reevaluated ages fully include uncertainties from the production rates and the recoil loss corrections, making them significantly larger.

The reevaluated CRE ages are within the (sometimes large) uncertainties identical to the ages originally given by Gyngard et al. (2009a, 2009b). However, this is just by coincidence. Since the new production rates for ${ }^{6,7} \mathrm{Li}$ are larger but the the new retention fractions are smaller, the net change is only very minor. For small grains, however, the differences are more pronounced because their age is dominated by the retention fraction, which is smaller using our model making the ages larger. For larger grains the new ages are lower than the 


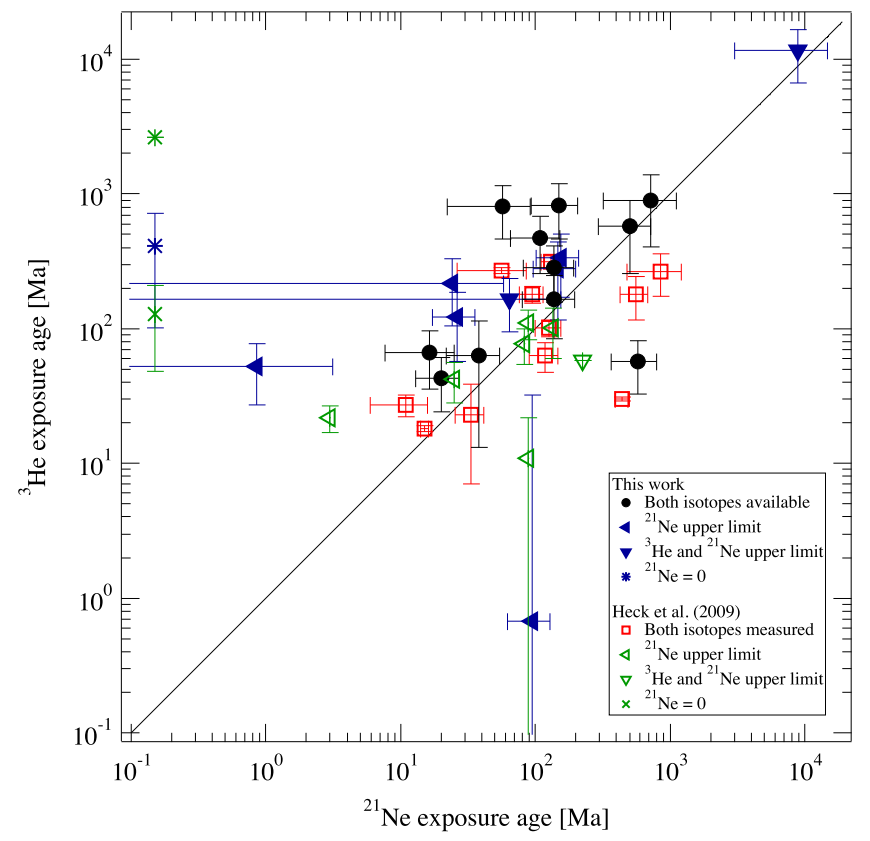

Figure 8. $T_{3}$ and $T_{21} \mathrm{CRE}$ ages. The cosmogenic ${ }^{3} \mathrm{He}$ and ${ }^{21} \mathrm{Ne}$ concentrations were measured by Heck et al. (2008, 2009). The straight line with slope 1 indicates identical $T_{3}$ and $T_{21}$ ages. Evaluations with a CRE age determined via cosmogenic ${ }^{21} \mathrm{Ne}$ of zero are shown randomly at $0.15 \mathrm{Ma}$ and marked with special symbols as described in the figure legend.

original ones due to the higher production rates modeled by us compared to the ones used by Gyngard et al. (2009a, 2009b). For those grains recoil losses are only minor and the differences in the retention fractions have only a minor effect on the ages.

Figure 7 shows that the CRE ages fall in a wide range from a few tens of Ma to more than $1 \mathrm{Ga}$. The two oldest grains have ages slightly older than $1 \mathrm{Ga}$, four grains have CRE ages between $100 \mathrm{Ma}$ and $1 \mathrm{Ga}$, and the two youngest grains have CRE ages $<100 \mathrm{Ma}$. The three oldest grains are all $\leqslant 5 \mu \mathrm{m}$ and the recoil correction was significant, which makes the ages relatively uncertain. However, within uncertainty all except for one ages are consistent with the grain survival theory, e.g., Jones et al. (1994, 1997).

\subsection{CRE Ages Based on ${ }^{3} \mathrm{He}$ and ${ }^{21} \mathrm{Ne}$}

In Figure 8 we compare the data data originally measured by Heck et al. (2009) and re-evaluated by Ott et al. (2009; red triangles) with the reevaluated CRE ages determined using our model (black dots). The newly calculated CRE ages and retention fractions are given in Table 4. In total Heck et al. (2009) measured 22 presolar $\mathrm{SiC}$ grains and found measurable helium and neon concentrations in 11 of them. One of the grains had no cosmogenic ${ }^{3} \mathrm{He}$ or ${ }^{21} \mathrm{Ne}$ concentration and is therefore not evaluated here. The values originally published by Heck et al. (2009) were reevaluated by Ott et al. (2009) using a slightly improved method for recoil correction and including the production and loss of tritium for ${ }^{3} \mathrm{He}$ production. Even after the improved recoil correction, the CRE ages based on ${ }^{3} \mathrm{He}\left(T_{3}\right)$ were older then the CRE ages based on ${ }^{21} \mathrm{Ne}\left(T_{21}\right)$ for five out of the 22 studied grains. Since we model even lower retention fractions for spallogenic ${ }^{3} \mathrm{He}$ we obtain even higher $T_{3}$ ages, therefore increasing the apparent discrepancy between $T_{3}$ and $T_{21}$. However, the situation is not as bad as it first looks. Since we give for the first time uncertainties for the production rates and the recoil losses, which are relatively large for ${ }^{3} \mathrm{He}$, we can conclude that expect for one grain $T_{3}$ and $T_{21}$ CRE ages agree within their $2 \sigma$ uncertainties (if ${ }^{3} \mathrm{He}$ and ${ }^{21} \mathrm{Ne}$ were measured). The one discrepant grain has a higher $T_{21}$ than $T_{3}$ age, which is most likely due to helium loss. For grains where one or both cosmogenic isotopes are given as upper limits, one grain has a ${ }^{21} \mathrm{Ne}$ record of zero and obviously $T_{3}$ and $T_{21}$ disagree. One grain has too little ${ }^{3} \mathrm{He}$, which can be explained by diffusion loss. For the other grains $T_{3}$ and $T_{21}$ agree within their $2 \sigma$ uncertainty. Note that for all of these grains, the cosmogenic ${ }^{21} \mathrm{Ne}$ is an upper limit with no uncertainties. The uncertainties given here for these grains are only from the production rate model. One grain has an extremely high CRE age of several billion years. Both, $T_{3}$ and $T_{21}$, ages are upper limits. The $T_{21}$ age was previously not calculated by Heck et al. (2009) since no recoil correction could be applied to such a small grain. Using the new recoil model we calculated the retention fraction for this grain with $1 \mu \mathrm{m}$ radius and determined an upper limit for the $T_{21}$ age as well.

Figure 9 shows the reevaluated CRE ages as a function of grain size. Two important conclusions can be drawn. First, there is no correlation between CRE ages and grains size. Second, the reevaluated CRE ages roughly agree with the calculated survival times, i.e., they are all smaller than the maximum survival times, as do the original ones.

\subsection{Additional Comments}

For studies of cosmogenic nuclides in presolar grains, three questions must be asked. First, can implantation of galactic ${ }^{3} \mathrm{He}$ affect the cosmogenic ${ }^{3} \mathrm{He}$ budget in the grain? Ott et al. (2009) proposed that implantation of low energetic helium particles from GCRs might (at least partly) explain why in some grains $T_{3}$ was higher than $T_{21}$. For the reevaluated data, however, $T_{3}$ and $T_{21}$ agree within the (relatively large) $2 \sigma$ uncertainties, therefore relaxing the requirement to explain apparent excess ${ }^{3} \mathrm{He}$. Though, ${ }^{3} \mathrm{He}$ implantation cannot be excluded and further studies are recommended.

Second, is the cosmogenic record in the grains affected by nuclide production in the meteorite? All presolar grains considered here are from the Murchison meteorite. Herzog et al. (1997) determined a (single stage) CRE age for Murchison of $1.8 \pm 0.3 \mathrm{Ma}$. Based on the cosmogenic production rate model by Leya \& Masarik (2009) we can estimate production rates for ${ }^{3} \mathrm{He}$ of $100 \times 10^{-10} \mathrm{~cm}^{3} \mathrm{STP} \mathrm{g}^{-1} \mathrm{Ma}^{-1}$ and for ${ }^{21} \mathrm{Ne}$ of $30 \times 10^{-10} \mathrm{~cm}^{3} \mathrm{STP} \mathrm{g}^{-1} \mathrm{Ma}^{-1}$ for $\mathrm{SiC}$ grains within the Murchison meteoroid, i.e., about three times and two times lower than the respective production rates in the ISM. With the CRE age for Murchison we can estimate that even for the grains with the shortest CRE ages nuclide production during meteorite transit contributes at best a few percent only, which is within the given uncertainties. This agrees well with the findings of Tang \& Anders (1988b).

Third, was there any nuclide production in the grains in the forming solar system? GCR irradiation of presolar grains in the solar nebula prior to their incorporation into the meteoroid parent bodies can be neglected for various reasons. During this stage GCRs in the disk are suppressed by several orders of magnitude due to an enhanced magnetic field of the protosun (e.g., Cleeves et al. 2013) and the midplane of the disk was 
Table 4

Reevaluated Retention Fractions and Grain Ages for the Presolar SiC Grains Measured by Heck et al. (2009)

\begin{tabular}{|c|c|c|c|c|c|}
\hline $\begin{array}{l}\text { Grain } \\
\text { Label }\end{array}$ & $\begin{array}{c}\text { Grain Radius } \\
(\mu \mathrm{m})\end{array}$ & $\begin{array}{c}\text { Retention } \\
\text { of }{ }^{3} \mathrm{He}\end{array}$ & $\begin{array}{c}T_{3} \\
(\mathrm{Ma})\end{array}$ & $\begin{array}{l}\text { Retention } \\
\text { of }{ }^{21} \mathrm{Ne}\end{array}$ & $\begin{array}{c}T_{21} \\
\text { (Ma) }\end{array}$ \\
\hline $\bar{L} 2-01$ & 3.65 & 0.081 & $809 \pm 348$ & 0.713 & $57 \pm 35$ \\
\hline L2-03 & 17.8 & 0.240 & $57 \pm 25$ & 0.929 & $574 \pm 211$ \\
\hline L2-05 & 2.85 & 0.067 & $412 \pm 311$ & 0.652 & $\sim 0$ \\
\hline L2-06 & 8.65 & 0.150 & $43 \pm 19$ & 0.862 & $20 \pm 7$ \\
\hline L2-07 & 4.40 & 0.093 & $1 \pm 32$ & 0.753 & $<94 \pm 33$ \\
\hline L2-10 & 4.50 & 0.094 & $217 \pm 112$ & 0.757 & $<24 \pm 34$ \\
\hline L2-11 & 5.90 & 0.115 & $164 \pm 81$ & 0.807 & $138 \pm 58$ \\
\hline L2-12 & 5.50 & 0.109 & $828 \pm 364$ & 0.795 & $149 \pm 56$ \\
\hline L2-13 & 3.90 & 0.082 & $337 \pm 165$ & 0.719 & $<153 \pm 54$ \\
\hline L2-14 & 5.50 & 0.109 & $473 \pm 213$ & 0.795 & $108 \pm 43$ \\
\hline L2-15 & 4.80 & 0.099 & $280 \pm 164$ & 0.770 & $<146 \pm 51$ \\
\hline L2-25 & 2.45 & 0.059 & $888 \pm 486$ & 0.611 & $722 \pm 401$ \\
\hline L2-27 & 1.00 & 0.027 & $<11592 \pm 4950$ & 0.320 & $<8944 \pm 5917$ \\
\hline $\mathrm{L} 2-57$ & 2.90 & 0.067 & $575 \pm 317$ & 0.656 & $504 \pm 210$ \\
\hline
\end{tabular}

Note. Grain labels and sizes are taken from Heck et al. (2009).

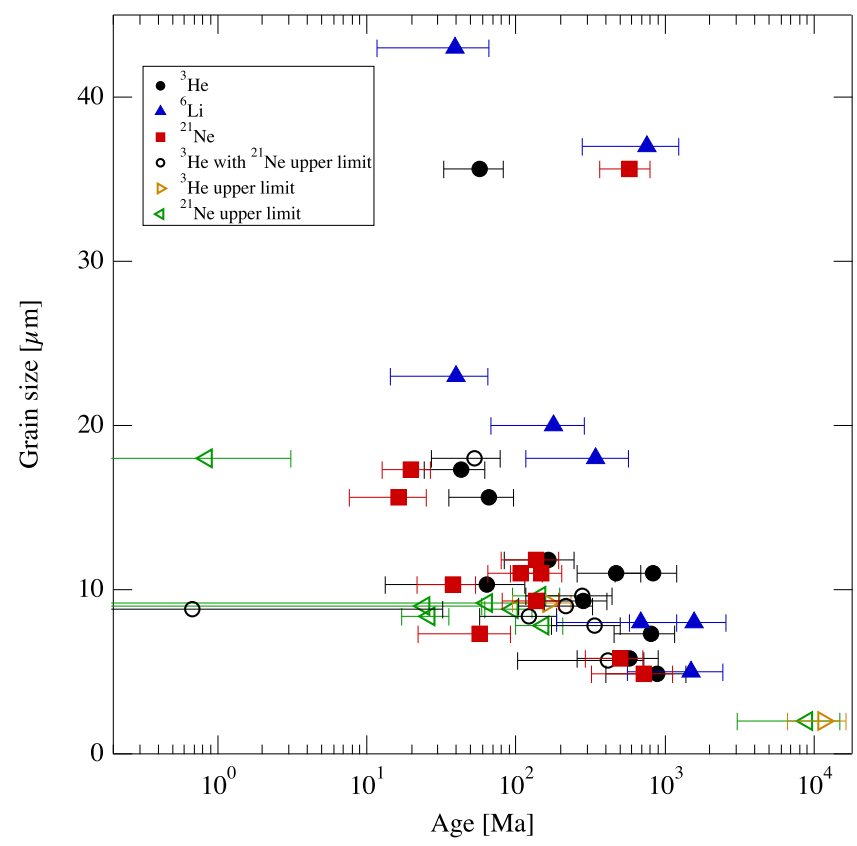

Figure 9. Reevaluated $T_{3}, T_{6}$, and $T_{21} \mathrm{CRE}$ ages as a function of grain size. Open symbols show $T_{3}$ and $T_{21}$ ages with upper limits in either one or both isotopes. All grains agree within uncertainty with the theoretical grain survival time of $\sim 600 \mathrm{Ma}$ (Jones et al. 1994, 1997).

shielded from SCRs by the thick envelope of gaseous hydrogen, e.g., Trappitsch \& Ciesla (2015).

\section{CONCLUSIONS}

We presented a purely physical model to calculate CRE ages of presolar $\mathrm{SiC}$ grains. The cosmogenic production rates are calculated using a state-of-the-art nuclear cross-section database and a GCR spectrum in the ISM consistent with recent
Voyager data (Stone et al. 2013). The recoil model, which is fully consistent with the production rate model, is based on TALYS-1.2 and INCL4.5/ABLA07 predictions and takes all incident energies for protons and $\alpha$ particles into account. The results for the recoil model compare well to experimental data.

Using the model we reevaluated the presolar grain data for ${ }^{3} \mathrm{He}$ and ${ }^{21} \mathrm{Ne}$ from Heck et al. (2008, 2009) and the lithium data from Gyngard et al. (2009a, 2009b). Unfortunately, the two studies were not performed on the same set of grains. There are two important findings. First, most CRE ages based on ${ }^{3} \mathrm{He}$ and ${ }^{21} \mathrm{Ne}$ agree within the (sometimes large) $2 \sigma$ uncertainties. Second, the CRE ages for most presolar grains are smaller than the predicted survival times. The results obtained are relatively robust because interferences from implanted low-energy GCRs into the presolar $\mathrm{SiC}$ grains and/or from cosmogenic production within the meteoroid can be neglected.

Correlated studies of helium, lithium, and neon isotopes in individual presolar grains would allow to better constrain their CRE ages because such combined studies would allow to better understand and quantify possible problems due to, e.g., contamination, implantation, and/or diffusion. Recently, Gyngard et al. (2014) reported preliminary results on lithium in presolar $\mathrm{SiC}$ grains. The same grains were subsequently analyzed for cosmogenic helium and neon and the preliminary evaluation for all cosmogenic nuclides was reported by Heck et al. (2015). Such combined studies could help to a better understand transport and survival processes of presolar grains in the ISM before their incorporation into the forming solar system.

We would like to thank an anonymous reviewer as well as Dieter H. Hartmann for their thorough reviews, which helped to significantly improve this manuscript. R.T. is supported by NASA Headquarters under the NASA Earth and Space Science Fellowship Program through grant NNX12AL85H and was 
partially supported by the NASA Cosmochemistry Program through grant NNX09AG39G (to A. M. Davis). I.L. is partly supported by the Swiss National Science Foundation (SNF).

\section{REFERENCES}

Ammon, K., Masarik, J., \& Leya, I. 2009, M\&PS, 44, 485

Bernatowicz, T. J., Messenger, S., Pravdivtseva, O., Swan, P., \& Walker, R. M. 2003, GeCoA, 67, 4679

Bernatowicz, T. J., \& Zinner, E. (ed.) 1997, in AIP Conf. Ser. 402, Astrophysical Implications of the Laboratory Study of Presolar Materials (Melville, NY: AIP)

Beyer, G.-J., \& Herrmann, E. 1978, Habilitation thesis, Technische Universität Dresden

Biersack, J. 1981, NucIM, 182/183, 199

Boudard, A., Cugnon, J., Leray, S., \& Volant, C. 2002, PhRvC, 66, 044615

Cameron, A. G. W., \& Truran, J. W. 1977, Icar, 30, 447

Castagnoli, G., \& Lal, D. 1980, Radiocarbon, 22, 133

Cleeves, L. I., Adams, F. C., \& Bergin, E. A. 2013, ApJ, 772, 5

Davids, C. N., Laumer, H., \& Austin, S. M. 1970, PhRvC, 1, 270

Foster, P. N., \& Boss, A. P. 1996, ApJ, 468, 784

Foster, P. N., \& Boss, A. P. 1997, ApJ, 489, 346

Greiner, D. E., Lindstrom, P. J., Heckman, H. H., Cork, B., \& Bieser, F. S. 1975, PhRvL, 35, 152

Gyngard, F., Amari, S., Zinner, E., \& Ott, U. 2009a, ApJ, 694, 359

Gyngard, F., Amari, S., Zinner, E., \& Ott, U. 2009b, PASA, 26, 278

Gyngard, F., Avila, J. N., Ireland, T. R., \& Zinner, E. 2014, LPSC, 45 abstract \#2348

Heck, P. R., Gyngard, F., Maden, C., et al. 2015, LPSC, 46 abstract \#1748

Heck, P. R., Gyngard, F., Meier, M. M. M., et al. 2008, LPSC, 39 abstract \#1239

Heck, P. R., Gyngard, F., Ott, U., et al. 2009, ApJ, 698, 1155

Herzog, G. F., Vogt, S., Albrecht, A., et al. 1997, M\&PS, 32, 413

Jones, A. P., Tielens, A. G. G. M., Hollenbach, D. J., \& McKee, C. F. 1994, ApJ, 433, 797

Jones, A. P., Tielens, A. G. G. M., Hollenbach, D. J., \& McKee, C. F. 1997, in AIP Conf. Ser. 402, Astrophysical implications of the laboratory study of presolar materials, ed. T. J. Bernatowicz, \& E. Zinner (Melville, NY: AIP), 595

Koning, A. J., Hilaire, S., \& Duijvestijn, M. C. 2008, in Proc. Int. Conf. Nuclear Data for Science and Technology, ed. O. Bersillon et al. (Nice: EDP Sciences) abstract \#058

Kruger, S. T., \& Heymann, D. 1973, PhRvC, 7, 2179

Lagarde-Simonoff, M., Regnier, S., Sauvageon, H., \& Simonoff, G. 1976, NuPhA, 260, 369

Lewis, R. S., Amari, S., \& Anders, E. 1990, Natur, 348, 293

Lewis, R. S., Amari, S., \& Anders, E. 1994, GeCoA, 58, 471

Leya, I. 1997, PhD thesis, Univ. Hannover

Leya, I., Begemann, F., Weber, H. W., Wieler, R., \& Michel, R. 2004, M\&PS, 39,367

Leya, I., \& Masarik, J. 2009, M\&PS, 44, 1061

Meier, M. M. M., Schmitz, B., Alwmark, C., et al. 2014, M\&PS, 49, 576

Meyer, B. S., \& Zinner, E. 2006, in Meteorites and the Early Solar System II, ed. D. S. Lauretta, \& H. Y. McSween (Tucson, AZ: Univ. Arizona Press)

Michel, R., Gloris, M., Lange, H.-J., et al. 1995, NIMPB, 103, 183

Mohapatra, R. K., Merchel, S., Ott, U., Herpers, U., \& Michel, R. 2001, LPSC, 32 abstract \#1296

Ott, U. 2001, P\&SS, 49, 763

Ott, U., Altmaier, M., Herpers, U., et al. 2001, M\&PSA, 36, A155

Ott, U., Altmaier, M., Herpers, U., et al. 2005, M\&PS, 40, 1635

Ott, U., \& Begemann, F. 2000, M\&PS, 35, 53

Ott, U., Heck, P. R., Gyngard, F., et al. 2009, PASA, 26, 297

Overholt, A. C., \& Melott, A. L. 2013, E\&PSL, 377, 55

Raisbeck, G. M., Lestringuez, J., \& Yiou, F. 1972, PhRvC, 6, 685

Reedy, R. C. 1989, LPSC, 20 abstract \#888

Simpson, J. A. 1983, ARNPS, 33, 323

Steinberg, E. P., \& Winsberg, L. 1974, PhRvC, 10, 1925

Stone, E. C., Cummings, A. C., McDonald, F. B., et al. 2013, Sci, 341, 150

Tang, M., \& Anders, E. 1988a, GeCoA, 52, 1245

Tang, M., \& Anders, E. 1988b, ApJL, 335, L31

Trappitsch, R., \& Ciesla, F. J. 2015, ApJ, 805, 5

Trappitsch, R., \& Leya, I. 2013, M\&PS, 48, 195

Ziegler, J. F. 2004, NIMPB, 219, 1027

Zinner, E. 2014, in Treatise on Geochemistry, ed. K. K. Turekian, \& H. D. Holland (2nd ed.; Oxford, UK: Oxford Univ. Press, Elsevier) 